\title{
A Q-Slot Monopole for UWB Body-Centric Wireless Communications
}

\author{
Bright Yeboah-Akowuah, Panagiotis Kosmas, Senior Member, IEEE, and Yifan Chen, Senior Member, IEEE
}

\begin{abstract}
This paper presents a novel and simple ultrawideband (UWB) printed rectangular monopole antenna (PRMA) for body-centric wireless communications. The design is based on etching a $Q$-slot on a rectangular radiator, and is optimized to produce the largest bandwidth in free space and close to the human body. We analyze the design of the proposed antenna and assess its performance in terms of bandwidth, gain, efficiency, and radiation patterns. We also characterize the antenna in the time-domain by calculating its fidelity factor. Our results show that the $Q$-slot antenna maintains its bandwidth when placed in close contact with the human body, or in contact with breast-mimicking tissue phantoms. The very good agreement between calculated and measured antenna performance in free space and on body suggests that the antenna is immune to variations in the human tissue, and is also robust to fabrication tolerances.
\end{abstract}

Index Terms-Ultra-wideband antenna, printed monopole, body-centric communications.

\section{INTRODUCTION}

$\mathbf{F}$ ollowing the release of the ultra-wideband (UWB) spectrum of 3.1-10.6 GHz for unlicensed use by the Federal Communications Commission (FCC) in U.S.A in February, 2002 [1], a lot of attention has been given to applications of UWB technology. Amongst these applications, bodycentric wireless communications involving body area networks (BANs) require devices to communicate off-, on-, and in-body. Significant progress in BANs has led to rapid development of UWB antennas for wearable applications in sports, military equipment, and biomedical engineering [1], [2].

The impact of UWB antennas on body-centric applications is becoming increasingly important due to the need for high data transmission systems with low power requirements. The advantages of UWB over narrowband antennas include large bandwidth, high data rate, resistance to interference, and low power consumption [2], [3]. A large absolute bandwidth also provides high resilience to fading and enhances the signal robustness for data transmission, which is an advantage for body-centric wearable units that operate in very challenging environments. However the design of UWB antennas is much more challenging than conventional broadband antennas, as UWB antennas require broadband operation in terms of

B. Yeboah-Akowuah and P. Kosmas are with the Department of Informatics, Faculty of Natural and Mathematical Sciences, King's College London, WC2R 2LS London, U.K. (e-mail: bright.yeboah-akowuah@kcl.ac.uk; panagiotis.kosmas@kcl.ac.uk).

Y. Chen is with the Faculty of Science and Engineering, Faculty of Computing and Mathematical Sciences, The University of Waikato, Hamilton 3240, New Zealand, and also with the Department of Electrical and Electronic Engineering, Southern University of Science and Technology, Shenzhen 518055, China. (e-mail: chen.yf@sustc.edu.cn). impedance matching, group delay, radiation patterns, and the ability to maintain the shape of the received signal.

Many UWB antennas for body-centric communications have been reported in the literature [4]-[6]. To achieve greater accuracy, antennas for body-centric communications must be designed in the presence of the human body, as body tissues absorb a great portion of the power supplied to the antenna and affect the antenna input impedance, radiation pattern and resonant frequency [3]. A relatively high efficiency of more than $50 \%$ in the vicinity of the human body is an important requirement for on-body applications, and can be achieved with cavity slot antennas with non-negligible height [7].

This paper presents an UWB printed rectangular monopole antenna (PRMA) [8] with attractive features (bandwidth, efficiency, high fidelity, high immunity and compactness), which make it suitable for body-centric communications. The proposed antenna has very stable performance when in close contact with the human body, in terms of input impedance, return loss, and radiation patterns across the UWB spectrum. The paper is structured as follows: Section II presents our antenna geometry and design, while Section III analyses the antenna performance in free space and on-body, comparing simulated and measured results. Conclusions are given in Section IV.

\section{ANTENNA DESIGN}

\section{A. Antenna Geometry}

The geometry of the proposed antenna consists of an $L_{s} \times W_{s}=36.6 \times 39 \mathrm{~mm}^{2}$ FR4 substrate of thickness $1.6 \mathrm{~mm}$ (with $\varepsilon_{r}=3, \tan \delta=0.01$ ). On top of the substrate is an $L \times W=18.3 \times 23.8 \mathrm{~mm}^{2}$ rectangular radiator. A thin Q-slot is etched on the radiator to generate additional resonance in the structure. At the back of the substrate is a partial rectangular ground plane measuring $36.6 \mathrm{xgr} \mathrm{mm} \mathrm{mm}^{2}$ as depicted in Fig.1. A $50 \Omega$ coaxial transmission line is used to feed the antenna.

\section{B. Design Process}

While PRMAs have been designed previously for wideband operation in the mobile and personal communications frequency range [8], operation in the UWB band is more challenging for a simple PRMA structure. UWB operation has been achieved with a printed circular disc monopole antenna [9], but the required substrate length $L_{s}=50 \mathrm{~mm}$ may be a limiting factor for on-body applications. Our design in Fig. 1 can be regarded as combining elements from these two previous designs (rectangular and circular), with additional design parameters that can be tailored to specific performance 


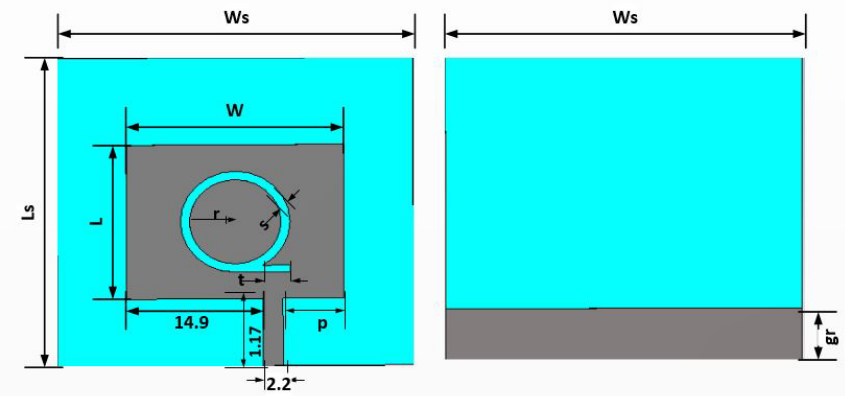

Fig. 1. Geometry of the proposed antenna: (left) top view, and (right) bottom view. All dimensions are in $\mathrm{mm}$.
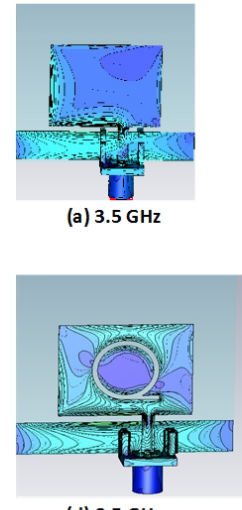

(d) $3.5 \mathrm{GHz}$

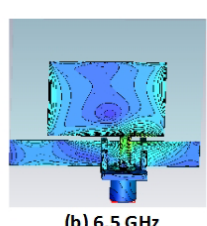

(b) $6.5 \mathrm{GHz}$

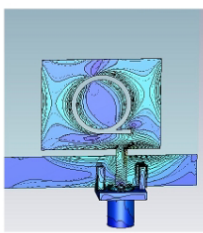

(e) $6.5 \mathrm{GHz}$
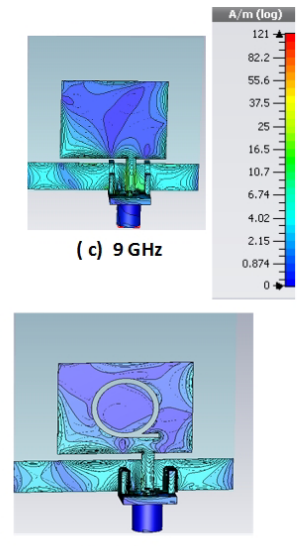

(f) $9 \mathrm{GHz}$
Fig. 2. Surface current densities PRMA (a-c) without Q-slot (d-f) with Q-slot.

requirements. In fact, the insertion of slots on radiators in order to enhance performance or achieve miniaturisation has been widely reported in the literature [10], [11]. For example, Uslots have been employed to achieve broadband operation in [12]-[14]. Our design proposes to use a Q-slot to enhance the monopole's performance over the UWB spectrum.

TABLE I

PARAMETERS OF THE ANTENNA SHOWN IN FIG. 1 (HEIGHT AND WIDTH OF THE FEED ARE FIXED AND SHOWN IN THE FIGURE)

\begin{tabular}{llc}
\hline \hline Parameter & Description & Value \\
\hline$L_{s}$ & substrate length & $36.6 \mathrm{~mm}$ \\
\hline$W_{s}$ & substrate width & $39.0 \mathrm{~mm}$ \\
\hline$L$ & radiator length & $18.3 \mathrm{~mm}$ \\
\hline$W$ & radiator width & $23.8 \mathrm{~mm}$ \\
\hline$h$ & substrate height & $1.6 \mathrm{~mm}$ \\
\hline$s$ & slot width & $1.0 \mathrm{~mm}$ \\
\hline$t$ & q-descender & $6.0 \mathrm{~mm}$ \\
\hline$p$ & feeding position & $6.7 \mathrm{~mm}$ \\
\hline$g_{r}$ & ground length & $6.3 \mathrm{~mm}$ \\
\hline$r$ & outer radius of the Q-slot & $6.0 \mathrm{~mm}$ \\
\hline \hline
\end{tabular}

In order to acquire a first estimate of the Q-slot's appropri-
TABLE II

PRMA METRICS WITH AND WITHOUT THE Q-SLOT

\begin{tabular}{lllllll}
\hline \hline Frequency[GHz] & 3.5 & 6.5 & 9.0 & 3.5 & 6.5 & 9.0 \\
\hline & \multicolumn{2}{l}{ With Q-slot } & \multicolumn{5}{c}{ Without Q-slot } \\
\hline Directivity[dBi] & 5.06 & 5.03 & 6.15 & 2.11 & 4.16 & 5.96 \\
\hline Radiation eff.[\%] & 98.40 & 99.60 & 98.68 & 95.60 & 96.87 & 97.30 \\
\hline Total eff.[\%] & 98.30 & 99.45 & 99.56 & 94.8 & 96.10 & 96.70 \\
\hline Realised gain[dB] & 4.33 & 4.90 & 6.0 & 3.14 & 3.90 & 5.61 \\
\hline \hline
\end{tabular}

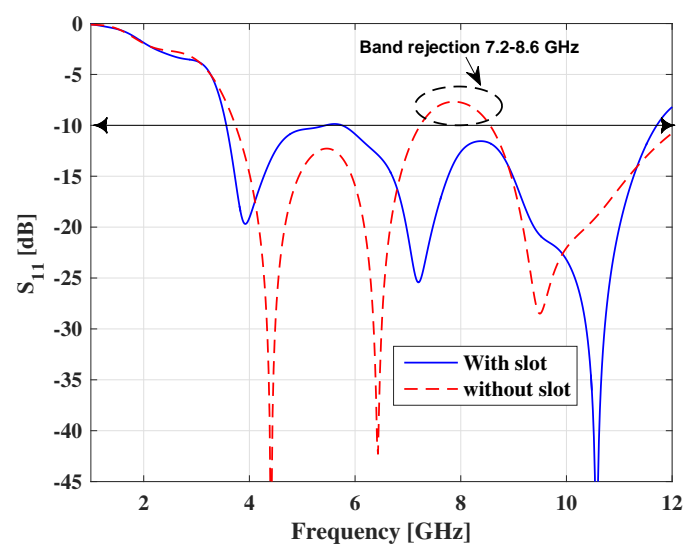

Fig. 3. Calculated reflection coefficient for the PRMA with and without the Q-slot. The introduction of the Q-slot retains $S_{11}$ under $-10 \mathrm{~dB}$ along the whole UWB spectrum.

ate dimensions, we studied surface current distributions and contours to identify the size of a relatively weak current area where the slot can be inserted. To this end, we modelled the antenna without the slot in CST Microwave Studio and plotted current distributions at representative frequencies of $3.5 \mathrm{GHz}$, $6.5 \mathrm{GHz}$ and $9 \mathrm{GHz}$, shown in Fig. 2. These plots reveal a relatively weak surface current density at the central part of the structure, and thus the inserted Q-slot's dimensions were chosen to fit that area. Then, the antenna parameters were optimised using a Trust Region Framework (TRF) algorithm within CST Microwave Studio to achieve the final design of Fig.1. The parameters for the antenna final design, which results in a reflection coefficient $S_{11}$ under $-10 \mathrm{~dB}$ from 3.5 to $11.7 \mathrm{GHz}$, are summarised in Table I.

The impact of inserting the slot on the antenna reflection coefficient $S_{11}$ is shown in Fig. 3, which demonstrates that inserting the slot can increase the distances between the antenna resonant frequencies and extend the bandwidth for which $S_{11}<-10 \mathrm{~dB}$ from 3.5 to $11.7 \mathrm{GHz}$. Moreover, inserting the slot results in an increase in the antenna's directivity, efficiency, and gain across the whole UWB spectrum, as shown in Table II for three representative frequencies.

\section{Parametric Study}

To evaluate the impact of some of the antenna design parameters defined in Table I, we performed a parametric study varying one of these parameters at a time, while keeping all others fixed to those of Table I. As our focus was on the impact 
of the slot, our study examined the effect of the following parameters with reference to Fig. 1: slot width $s$, outer radius of the slot $r$, Q-descender $t$, and feeding position $p$.

Fig. 4 plots the antenna's reflection coefficient $S_{11}$ as function of each of the four parameters. For all four parameters under study, our final choices resulted in the broadest bandwidth for $S_{11}$ under $-10 \mathrm{~dB}$ (red solid line). It is clear from these plots that all parameters have significant impact on the antenna return loss. Changing the position of the feed $p$ affects both the strength and location of the resonant frequencies as well as the frequency range where $S_{11}<-10 \mathrm{~dB}$, with $p=6.7 \mathrm{~mm}$ and $p=0 \mathrm{~mm}$ producing the broadest bandwidths (see Fig. 4(a)). Relative to our finalized design, changes in the Q-slot parameters $r, s$ and $t$ can improve matching, (i.e. lower the $S_{11}$ ) in the range of $4.0-6.0 \mathrm{GHz}$, but result in a lower bandwidth for which $S_{11}<-10 \mathrm{~dB}$, as indicated in Fig. 4(b), (c), (d) by comparing the various lines to the red solid line of the final design.

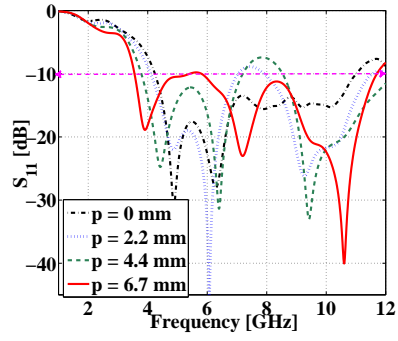

(a)

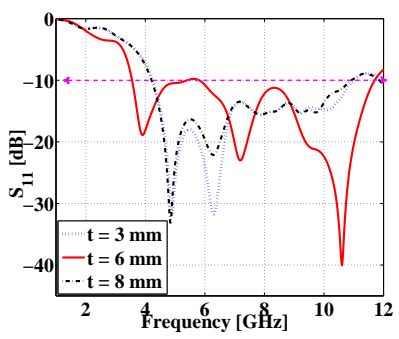

(c)

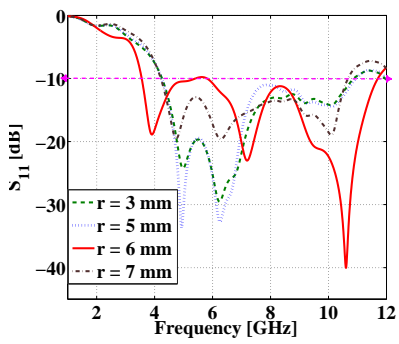

(b)

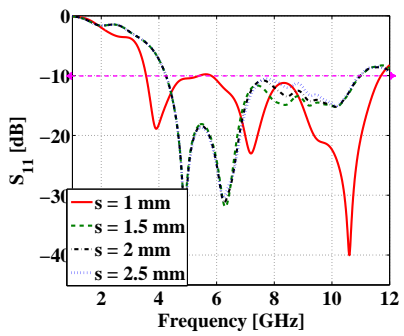

(d)
Fig. 4. Impact of design parameters $p, r, t$ and $s$ on $S_{11}$ by varying one parameter at a time, with respect to (a) feeding position $p$, (b) slot radius $r$, (c) Q-descender $t$, and (d) slot width $s$. The finalized parameters correspond to the (red) solid line in all plots.

We also examined the effect of these parameters on antenna efficiency, directivity, and gain, by calculating their values for the same parametric study as for $S_{11}$ in Fig. 4. In terms of efficiency, changing the Q-descender $t$ from its optimized value drops the efficiency significantly in the range up to 6 $\mathrm{GHz}$, and the same applies for the case of slot width $s=2.5$ $\mathrm{mm}$. For all other cases, the efficiency does not vary much from the values of the final design presented in Table II. The directivity and gain follow the same trends, and are in general optimal for the final design (see values in Table II). More specifically, the Q-descender $t$ affects the gain only when its length increases significantly $(t=8 \mathrm{~mm})$, while varying the slot width $s$ has little effect overall. Varying the outer radius of the slot $r$ can reduce the gain by $1-1.5 \mathrm{~dB}$ away from its optimal value at $9 \mathrm{GHz}$, but can also increase it slightly at lower frequencies. Varying the the feeding position $p$ has the most pronounced effect, with the optimal positions being $p=6.7$ and $0 \mathrm{~mm}$ (at the edge of the radiator).

\section{PERFORMANCE IN AIR AND ON-BODY}

\section{A. Free Space Return Loss}

To study the antenna operation in free space, we first compared its calculated (using CST) and measured reflection coefficient $S_{11}$. Fig. 5 shows the prototype of the proposed antenna which was experimentally characterised by a M9375A vector network analyser (Keysight). The antenna $S_{11}$ was calculated between $2-12 \mathrm{GHz}$, as this was the initial UWB spectrum of interest. However, our measurements showed that the antenna $S_{11}$ is under $-10 \mathrm{~dB}$ for frequencies up to $20 \mathrm{GHz}$. As shown in Fig. 6, the free space simulated and measured results agree very well up to $10 \mathrm{GHz}$, with some differences arising around the third resonance above $10 \mathrm{GHz}$, which is not as strong and narrow for the experimental measurement as for our simulation.

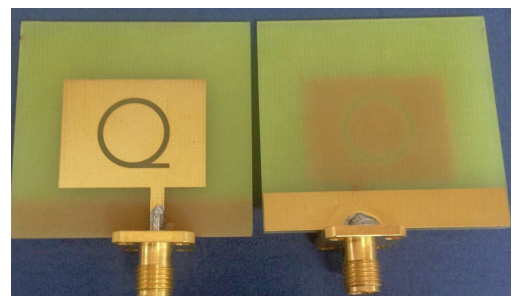

Fig. 5. Fabricated prototype used in our measurements.

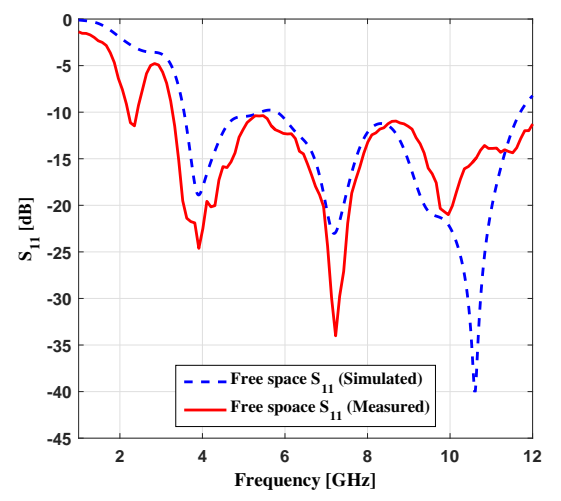

Fig. 6. Free space simulated (dashed) and measured (solid) $S_{11}$ of the proposed antenna in the frequency range up to $12 \mathrm{GHz}$.

\section{B. On-body Return Loss}

To study the antenna performance for on-body applications, we considered a three-layer phantom composed of wet skin $\left(\varepsilon_{r}=41.982, \sigma=2.0168\right)$, fat $\left(\varepsilon_{r}=5.2138, \sigma=0.13497\right)$ and muscle $\left(\varepsilon_{r}=51.936, \sigma=2.2216\right)$ calculated at the lower-edge band of UWB spectrum, 3.1 GHz [15]. We note that this is only an approximate tissue model, since the tissue properties are not constant across the whole frequency range. 
The dimensions of the phantom (50 $\mathrm{mm}$ x $50 \mathrm{~mm}$ x $400 \mathrm{~mm})$ are equivalent to a human arm model considered in [16], [17] as shown in Fig. 7(a). Using this model, we calculated the antenna $S_{11}$ when placed on or very close to the human body. The separation gap between the antenna and the phantom was varied between $3 \mathrm{~mm}$ to $15 \mathrm{~mm}$, and the calculated $S_{11}$ for the various positions is plotted in Fig. 7(b). The plots in Fig 7(b) suggest that the antenna does not detune even as close as $3 \mathrm{~mm}$ away from the human body.

To test this important observation experimentally, we carried out on-body measurements where we attached the antenna parallel onto the chest of a male human volunteer (age = 35 years, weight $=81 \mathrm{~kg}$, height $=172 \mathrm{~cm}, \mathrm{BMI}=27$ ) in upright position. We measured the $S_{11}$ with the VNA as the distance from the chest varied from 3 to $15 \mathrm{~mm}$. In addition to the chest measurements, we carried out direct onbody measurements on different parts of the body such as the arm and the wrist. The results from the chest measurements are plotted in Fig.8(a), where it is shown that the antenna maintains its UWB operation in the presence of the human body. Moreover, the measured on-body data for different body parts plotted in Fig. 8(b) shows an extended bandwidth from $3.1 \mathrm{GHz}$ to $20 \mathrm{GHz}$, for which $S_{11}$ is under $-10 \mathrm{~dB}$.

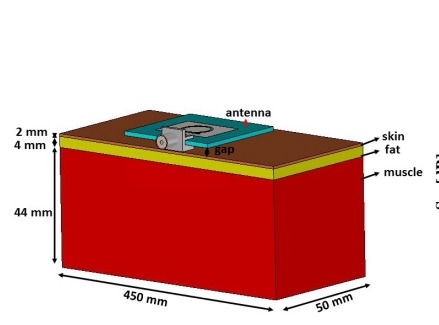

(a)

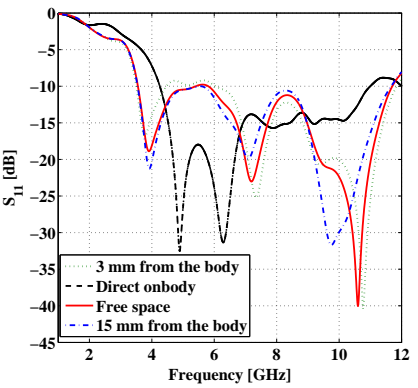

(b)
Fig. 7. Simulated on-body performance of the proposed antenna. (a) Antenna and three-layer phantom model for on-body simulations (b) Corresponding calculated return loss for various values of the gap between the antenna and the phantom.

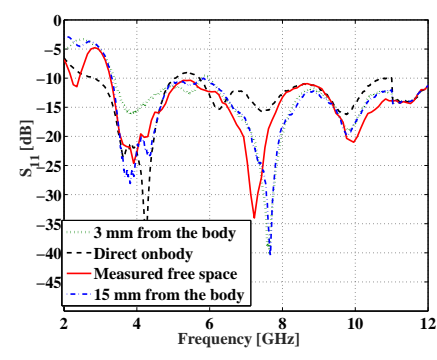

(a)

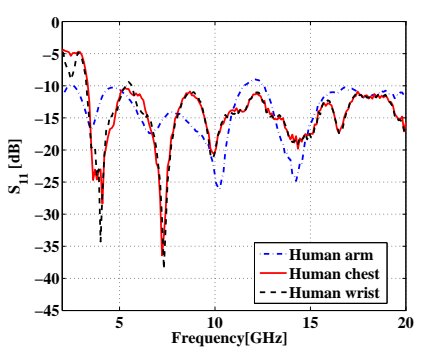

(b)
Fig. 8. (a) Measured $S_{11}$ when the antenna was placed on human subject with the same gap distances from his chest as in the simulations of Fig. 7 (the solid red lines is the measured $S_{11}$ reference in air). (b) Direct on-body measured $S_{11}$ (up to $20 \mathrm{GHz}$ ) for different parts of the body.

Using the developed simulation model of Fig. $7 b$ (a) with the antenna placed $3 \mathrm{~mm}$ away from the skin, we also calculated the specific absorption rate (SAR) for the antenna when placed on-body in order to calculate the maximum power that the antenna can transmit within safety limits. In accordance to
TABLE III

COMPOSITION OF TISSUE-MIMICKING MATERIALS. ABBREVIATIONS: V : VOLUME, W :WEIGHT, A: P-TOLUIC ACID, B: N-PROPANOL, C:GELATIN, D: FORMALDEHYDE, E: OIL, F: SURFACTANT, G :WATER. [19]

\begin{tabular}{llllllll}
\hline v\% oil & w\%A & w\%B & w \% C & w\% D & w\% E & w \% F & w \% G \\
\hline 50 & 0.045 & 1.81 & 7.69 & 0.18 & 44.42 & 2.89 & 42.96 \\
\hline
\end{tabular}

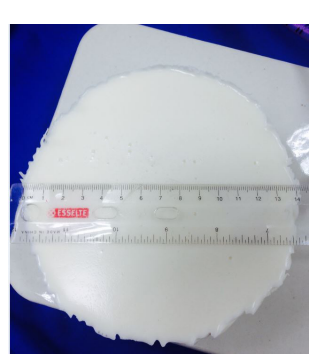

(a)

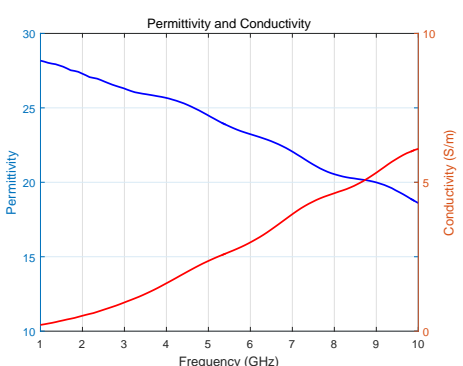

(b)

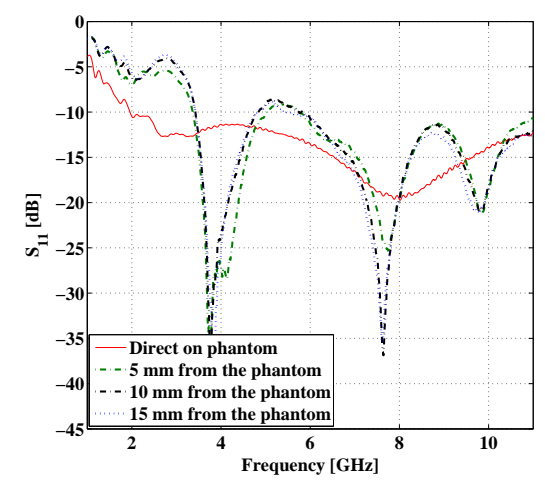

(c)

Fig. 9. Summary of our breast phantom measurements. (a) Photo of the homogeneous breast phantom; (b) Plot of its tissue-mimicking dielectric properties vs frequency; (c) Measured $S_{11}$ when the antenna is placed in close (varying) distances to the phantom.

the IEEE C95.1-2005 standard, the SAR average value over $10 \mathrm{~g}$ of human tissue mass should not exceed $2 \mathrm{~W} / \mathrm{Kg}$ [18]. When the antenna was fed with a $1 \mathrm{~W}$ peak input reference power, we calculated the SAR distribution averaged over 10 $g$ of human tissue mass, which reached a $15 \mathrm{~W} / \mathrm{Kg}$ value at $9.0 \mathrm{GHz}$. Thus, the delivered power of the antenna must be decreased to $133 \mathrm{~mW}$ in order to satisfy the strictest regulatory requirements by IEEE C95.1-2005. Considering the US FCC standard (1.6 W/ Kg limit), the corresponding peak SAR value was calculated at $38.1 \mathrm{~W} / \mathrm{Kg}$, which means that the delivered power must be reduced to $41.9 \mathrm{~mW}$ to stay below the 1.6 $W / K g$ limit.

\section{Operation in the presence of a breast phantom}

We also performed experiments to evaluate the antenna performance when placed in close proximity to a phantom mimicking average breast tissue properties [19], motivated by the current development of various medical diagnostic applications based on microwave technologies [20]. Our experiments are summarised in Fig. 9. The breast phantom was prepared from the materials listed on Table III, which 


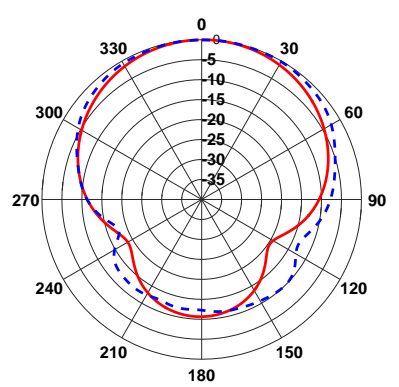

(a) $3.1 \mathrm{GHz}$

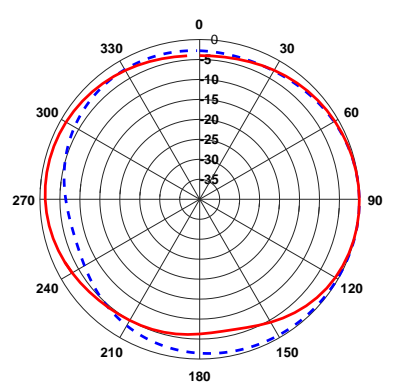

(d) $3.1 \mathrm{GHz}$

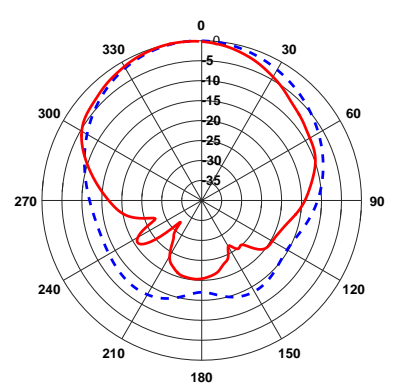

(b) $6.5 \mathrm{GHz}$

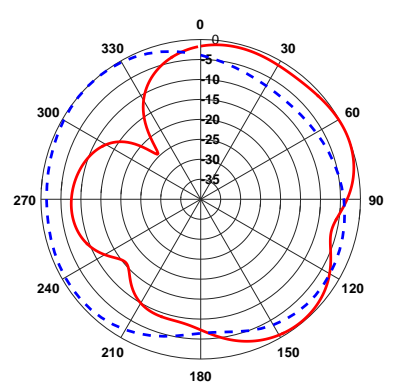

(e) $6.5 \mathrm{GHz}$

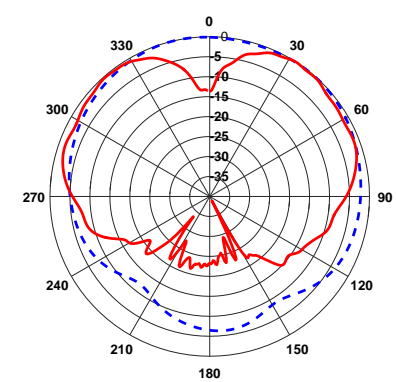

(c) $9 \mathrm{GHz}$

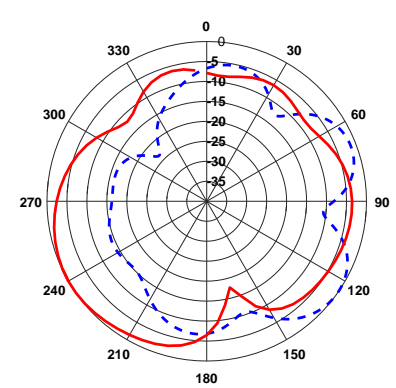

(f) $9 \mathrm{GHz}$

Fig. 10. Calculated (dotted line) and measured (solid line) radiation patterns when the antenna is placed on-body at $3.5 \mathrm{GHz}, 6.5 \mathrm{GHz}$ and $9 \mathrm{GHz}$ (a-c) azimuthal plane, (d-f) elevation plane.

shows the percentages by weight of all the components. The hemispherical breast phantom had a diameter of $14 \mathrm{~cm}$ and a depth of $10 \mathrm{~cm}$ (see Fig. 9(a)). The measured permittivity and conductivity values of the constructed breast phantom tissue are plotted vs. frequency in Fig. 9(b) for the UWB spectrum. The measured $S_{11}$ for the antenna placed on the breast phantom is plotted in Fig. 9(c). Although the properties of the tissue mimicking phantom are quite different from the simulated phantom or its equivalent tissue volume seen by the antenna when placed on-body as in Figs. 7 and 8, Fig. 9(c) suggests that the antenna is robust to variation in the properties of tissues and maintains its UWB operation.

\section{Radiation patterns}

The radiation patterns of the antenna in the azimuthal and elevation planes were evaluated both in free space and on-body at three representative frequencies of $3.5 \mathrm{GHz}, 6.5 \mathrm{GHz}$ and 9 GHz. Fig. 10 shows the on-body simulated and measured radiation patterns at azimuthal and elevation planes and the aforementioned frequency points. In the azimuthal plane, the antenna is more directive at lower frequencies, and the front to back ratio is approximately $20 \mathrm{~dB}$ due to tissue losses. At higher frequencies (e.g. $9 \mathrm{GHz}$ ), the patterns are quasiomnidirectional. In the elevation plane, the radiation patterns at the selected frequencies are more or less omnidirectional. There is good agreement in all cases between the simulated and measured on-body patterns, as suggested by the plots at the selected frequencies.

\section{E. Time-domain study}

An UWB antenna cannot be fully characterized without studying its time-domain operation. An important feature to study in this respect is the antenna's fidelity, which is a measure of its ability to preserve the shape of the pulse used to excite the antenna. To study the fidelity of the proposed antenna, we simulated its operation excited by a modulated Gaussian pulse given by,

$$
V_{\text {mod }}(t)=\left[\sin \left(2 \pi f_{c} t\right)\right] e^{-\left(\frac{t-d}{b}\right)^{2}}
$$

where $b$ is the pulse width, $d$ is the time delay, and $f_{c}$ is the modulated frequency. In order to satisfy FCC's strict emission limits on UWB antennas and at the same test fidelity across the whole UWB spectrum, we performed calculations for sinemodulated input signals centered at two different modulation frequencies, $f_{c}=4.5 \mathrm{GHz}$ and $6.5 \mathrm{GHz}$, using $b=220 \mathrm{ps}$, and $d=180 \mathrm{ps}$ in (1). Fig. 11 shows the modulated excitation signals and their corresponding power spectral density (PSD) with the emission mask, which confirms that these pulses are within FCC regulations.

The antenna fidelity can be computed from the crosscorrelation of the input excitation pulse and the output signal transmitted by the antenna after normalisation [21]. The 
fidelity factor $F$ can be expressed mathematically as,

$$
F=\max _{\tau}\left[\frac{\int_{-\infty}^{+\infty} x(t) \cdot y(t-\tau) \cdot d t}{\sqrt{\int_{-\infty}^{+\infty}|x(t)|^{2} \cdot d t \int_{-\infty}^{+\infty}|y(t)|^{2} \cdot d t}}\right]
$$

where $x(t)$ and $y(t)$ are the input and output signals, and $\tau$ is the time delay that maximizes (2). To calculate (2), we used field probes in CST that were placed at the far field of the transmitting antenna and recorded the radiated signals. The signals were cross-correlated using CST Microwave Studio and examples of these cross-correlations are shown in Fig. 12 for two different directions, $\left(\theta=0^{\circ}, \phi=0^{\circ}\right)$, and $(\theta=$ $\left.0^{\circ}, \phi=90^{\circ}\right)$. The fidelity factors for these directions were calculated to be $90.0 \%$ and $91.3 \%$, which can ensure minimal input pulse distortion [22].

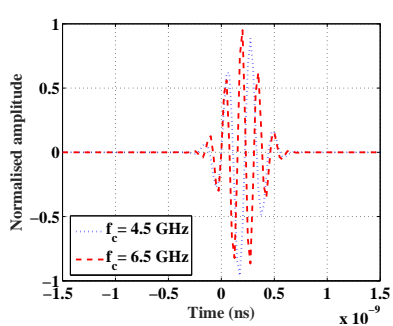

(a)

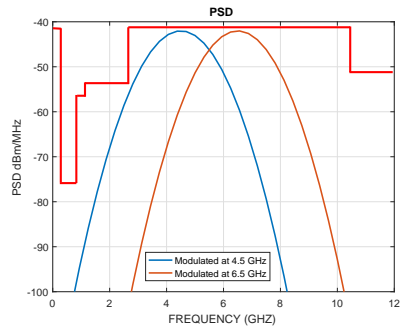

(b)
Fig. 11. (a) The modulated Gaussian pulse at 4.5 and $6.5 \mathrm{GHz}$, and (b) its corresponding power spectral density which is contained within the FCC mask.

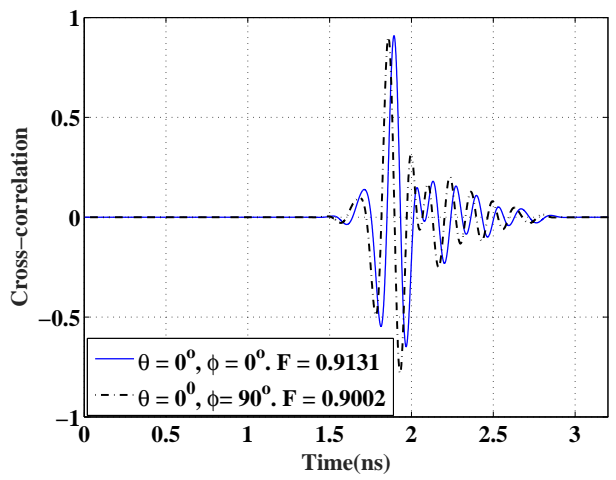

Fig. 12. Cross-correlation of the input pulse with the signal transmitted by the antenna as recorded for two different directions noted in the plot. Using (2), the fidelity factors for these directions were calculated to be $90.0 \%$ and $91.3 \%$.

\section{CONCLUSION}

This paper presents a novel printed rectangular monopole antenna (PRMA) suitable for body-centric applications. We evaluated the performance of the antenna in free space and onbody, and observed that the proposed antenna did not detune in close proximity of human body. We also demonstrated very good agreement between the simulated and measured return loss and radiation patterns for both free space and on-body scenarios. The antenna performs well in close contact with the human body with appreciable gains, radiation patterns and efficiency of $98 \%$ in free space and $52 \%$ on the body. Finally, we have also shown that the antenna could be a good candidate for UWB microwave breast cancer detection applications, by validating its UWB operation in experiments with a homogeneous phantom with dielectric properties in the range of normal (dense) breast tissue.

\section{ACKNOWLEDGMENT}

This work has been partly supported by COST Action TD1301, MiMed, through a short scientific mission to South University of Science and Technology of China. This work was also financially supported in part by Innovate UK under Grant No. 131750, and also in part by The Shenzhen Development and Reform Commission Funds under Grant [2015]944, Grant [2015]1939, and the Shenzhen Science, Technology and Innovation Commission Funds under Grant KQCX2015033110182368.

\section{REFERENCES}

[1] P. S. Hall and Y. Hao, Antenna and Propagation for Body-Centric Wireless Communications. Norwood, MA, USA, Artech House, 2006.

[2] J. Volakis, Antenna Engineering Handbook. McGraw-Hill, fourth ed., 2007.

[3] D. Valderas, J. I. Sancho, D. Puente, C. Ling, and X. Chen, Ultrawideband Antennas: Design and Applications. Imperial College Press, London, UK, 2011.

[4] A. Alomainy, Y. Hao, X. Hu, C. G. Parini, and P. S. Hall, "UWB on-body radio propagation and system modelling for wireless bodycentric networks," IEE Proc., Communications, vol. 153, pp. 107-114, Feb 2006.

[5] T. See and Z. N. Chen, "Experimental characterization of UWB antennas for on-body communications," IEEE Trans. Antennas and Propag., vol. 57, pp. 866-874, April 2009.

[6] M. M. Khan, Q. H. Abbasi, A. Alomainy, Y. Hao, and C. Parini, "Experimental characterisation of ultra-wideband off-body radio channels considering antenna effects," IET Micro., Antennas Propag., vol. 7, pp. 370-380, April 2013.

[7] N. Haga, K. Saito, M. Takahashi, and K. Ito, "Characteristics of cavity slot antenna for body-area networks," IEEE Trans. Antennas and Propag., vol. 57, pp. 837-843, April 2009.

[8] K. P. Ray and Y. Ranga, "Printed rectangular monopole antennas," in Proc. IEEE AP-S Internat. Symp., pp. 1636-1639, 2006.

[9] J. Liang, C. C. Chau, X. Chen, and C. G. Parini, "Printed circular disc monopole antenna for ultra-wideband applications," Electron. Lett., vol. 40, pp. 1246-1248, Sept. 2004.

[10] R. Bhalla and L. Shafai, "Broadband patch antenna with a circular arc shaped slot," in IEEE AP-S Internat. Symp., 2002.

[11] B. Yeboah-Akowuah, E. Kallos, G. Palikaras, Y. Chen, and P. Kosmas, "A novel compact planar inverted-F antenna for biomedical applications in the MICS band," in Proc. 8th EuCAP, Hague, Netherlands, pp. 822824, April 2014.

[12] K. F. Lee, S. Yang, A. Kishk, and K. M. Luk, "The versatile U-slot patch antenna," IEEE Trans. Antennas and Propag. Mag., vol. 52, pp. 71-88, Feb 2010.

[13] S. Weigand, G. Huff, K. Pan, and J. Bernhard, "Analysis and design of broad-band single-layer rectangular U-slot microstrip patch antennas," IEEE Trans. Antennas and Propag., vol. 51, pp. 457-468, March 2003.

[14] A. Deshmukh and K. Ray, "Analysis of broadband variations of U-slot cut rectangular microstrip antennas," IEEE Trans. Antennas and Propag. Mag., vol. 57, pp. 181-193, April 2015.

[15] "Calculation of the dielectric properties of body tissues.Institute for applied physics italian national research council." Website. http://niremf. ifac.cnr.it/tissprop.

[16] C.-H. Lin, K. Saito, M. Takahashi, and K. Ito, "A compact planar inverted-F antenna for $2.45 \mathrm{GHz}$ on-body communications," IEEE Trans. Antennas and Propag., vol. 60, pp. 4422-4426, Sept 2012. 
[17] B. Yeboah-Akowuah, E. Kallos, G. Palikaras, Y. Chen, and P. Kosmas, "A low profile microstrip patch antenna for body-centric communications at $2.45 \mathrm{GHz}$ band," in Proc. 9th EuCAP, Lisbon, Portugal, pp. 1-3, April 2015.

[18] "IEEE standard for safety levels with respect to human exposure to radio frequency electromagnetic fields, $3 \mathrm{kHz}$ to $300 \mathrm{GHz}$ amendment 1: Specifies ceiling limits for induced and contact current, clarifies distinctions between localized exposure and spatial peak power density," IEEE Std C95.1a-2010 (Amendment to IEEE Std C95.1-2005), pp. 1-9, March 2010

[19] M. Lazebnik, E. L. Madsen, G. Frank, and S. C. Hagness, "Tissue mimicking phantom and materials for narrowband and ultrawideband microwave applications," Phys.Med.Biol., vol. 50, pp. 4245-4258, Aug. 2005.

[20] Y. Chen, P. Kosmas, and S. Martel, "A feasibility study for microwave breast cancer detection using contrast-agent-loaded bacterial microbots," Internat. J. Antennas Propagat., vol. 309703 (11 pages), pp. 1246-1248, 2013.

[21] D. Lamensdorf and L. Susman, "Baseband-pulse-antenna techniques," IEEE Antennas and Propag. Mag., vol. 36, pp. 20-30, Feb 1994.

[22] G. Quintero, J. F. Zurcher, and A. K. Skrivervik, "System fidelity factor: A new method for comparing UWB antennas," IEEE Trans. Antennas and Propag., vol. 51, pp. 457-468, March 2003. 